\title{
Asymptomatic Cervical or Thoracic Lesions in Elderly Patients who Have Undergone Decompressive Lumbar Surgery for Stenosis
}

\author{
Boo Seop Kim¹, Jinsoo Kim¹, Han-Sang Koh, \\ Song Yup Han², Dong-Yeob Lee ${ }^{3}$, Kyeong Hwan Kim \\ ${ }^{1}$ Department of Orthopaedic Surgery, Hyundae Hospital, Namyangju, Korea \\ 2Department of Neurosurgery, Hyundae Hospital, Namyangju, Korea \\ ${ }^{3}$ Department of Neurosurgery, Wooridul Spine Hospital, Seoul, Korea
}

Study Design: A retrospective study.

Purpose: To evaluate the prevalence and risk factors of asymptomatic cervical or thoracic lesions in elderly patients who have undergone surgery for lumbar spinal stenosis.

Overview of Literature: Concurrent multiple spinal lesions have been reported in many studies with a varied prevalence, and described the characteristics of the disease and its treatment options. However, the cervical or thoracic lesions without apparent symptoms in patients with symptomatic lumbar stenosis had not been evaluated.

Methods: A total of 101 elderly patients (aged 65 or more), who had undergone surgery for lumbar spinal stenosis from January 2005 to December 2005, were enrolled in this study. All patients underwent lumbar magnetic resonance imaging (MRI) along with T2-weighted cervical and thoracic sagittal MRI prior to surgery. The concurrent cervical or thoracic lesions were classified according to the disease entity, and the severity of the lesions was graded from grade 0 (no lesion) to grade 4 (any lesion compressing the cord with a signal change). The prevalence of concurrent cervical and thoracic lesions was then analyzed. In addition, the risk factors for the development of concurrent lesions were evaluated, and the risk factors affecting the severity of the concurrent lesion were analyzed individually.

Results: Seventy-seven (76.2\%) and 30 (29.7\%) patients had a concurrent cervical and thoracic lesion, respectively. Twentysix patients $(25.7 \%)$ had both a cervical and thoracic lesion. There was a positive correlation between the symptom duration of lumbar stenosis and the prevalence of both cervical ( $p=0.044$ ) and thoracic $(p=0.022$ ) lesions.

Conclusions: The incidence of asymptomatic cervical or thoracic lesions is apparently high in elderly patients who have undergone surgery for lumbar spinal stenosis, particularly in those with longer symptom duration. This highlights the need for a preoperative evaluation of the cervical and thoracic spine in these patients.

Key Words: Asymptomatic, Concurrent, Cervical, Thoracic, Lumbar stenosis, Aged

\section{Introduction}

Spinal stenosis, of which the main cause is the progres- sive narrowing of the spinal canal through a degenerative process, is a common disease in elderly patients. Stenosis of the spinal column has a range of symptoms, such as claudication, radiculopathy and myelopathy, according to the

Received Jan 6, 2010; 1st Revised Jan 28, 2010; 2nd Revised Feb 1, 2010; 3rd Revised Feb 1, 2010; Accepted Feb 2, 2010

Corresponding author: Kyeong Hwan Kim, MD

Department of Orthopaedic Surgery, Hyundae Hospital, 663 Janghyeon-ri, Jinjeop-eup, Namyangju 472-865, Korea

Tel: +82-31-572-9118, Fax:+82-31-572-1194, E-mail: ponticuli@naver.com 
level and severity of the stenosis. Although spinal stenosis can occur at any level, it occurs most often at the most mobile segments of the spine, i.e., at the cervical and lumbar levels [1]. Most cases of spinal stenosis occur at single anatomical segments, such as the cervical, thoracic or lumbar segments. However, it can also develop simultaneously in multiple anatomical segments, which can create confusion in the diagnosis and treatment [2]. Teng and Papatheodorou [3] first described concurrent cervical and lumbar stenosis, and Dagi et al. [4] were the first to use the term "tandem spinal stenosis" to describe combined stenosis.

Some clinical studies reported tandem stenosis with a varied prevalence, and described the characteristics of the disease and its treatment options [1,2,4-8]. However, the diagnosis and definition of multiple concurrent spinal stenosis have yet to be clearly defined. Lee et al. [9] reported the prevalence of cervical and lumbar bony stenosis in their cadaveric skeleton study, as well as the prevalence of tandem cervical or thoracic bony stenosis. However, their study did not consider soft-tissue pathology, and could not clarify the relationship of the disease with the clinical symptoms, as they used cadavers. To date, the cervical or thoracic lesions without apparent symptoms in patients with symptomatic lumbar stenosis had not been evaluated. The awareness of the asymptomatic cervical or thoracic lesion before lumbar surgery is clinically important because the symptoms related to cord compression can emerge due to aggravation by a cervical or thoracic lesion through a prolonged flexion or extension position during surgery $[10,11]$.

The purpose of this retrospective study was to identify (1) the prevalence and risk factors of an asymptomatic cervical or thoracic lesion, and (2) the risk factors affecting the severity of the lesion at the cervical and thoracic spine in elderly patients who had undergone surgery for lumbar stenosis.

Table 1. Demographic data

\begin{tabular}{llc}
\hline \hline Parameters & & \\
\hline No. of patients & & 101 \\
& Male & 39 \\
& Female & 62 \\
Mean age (yr) & $71(65-86)$ \\
Symptom duration (mo) & $23(1.5-120)$ \\
Concurrent lesion level & \\
$\quad$ Cervical lesion (\%) & 76.2 \\
$\quad$ Thoracic lesion (\%) & 29.7 \\
Cervical and thoracic lesion $(\%)$ & 25.7 \\
\hline
\end{tabular}

\section{Materials and Methods}

A total of 101 consecutive elderly patients (aged 65 or more), who had undergone surgery for lumbar spinal stenosis within the period from January to December 2005, were enrolled in this study. There were 39 males and 62 females with a mean age of 71 years (range, 65 to 86 years) (Table 1). The mean symptom duration was 23 months (range, 1.5 to 120 months). Back and leg pain, with or without neurogenic claudication, was their chief complaint. A small number of patients reported mild axial neck pain or a tingling sensation of the upper extremities but the symptoms were not apparent. Mild symptoms were defined as symptoms showing less than 3 points on a visual analogue scale and did not disturb their daily life. All patients underwent lumbar magnetic resonance imaging (MRI) along with T2weighted cervical and thoracic sagittal MRI before surgery. Any patient with degenerative spondylolisthesis or instability was excluded. An orthopedic surgeon and a neurosurgeon, who were not involved in the surgery, evaluated all the radiological images using digitalized tools in a PACS system PiView 1.0 (Infinitt Co, Ltd., Seoul, Korea). Two surgeons classified the lesions and graded their severity on the T2-weighted cervical and thoracic sagittal MRI. The lesions were classified into a herniated intervertebral disc, ossified posterior longitudinal ligament, ossified ligamentum flavum and bony stenosis. The severity of the lesions was graded as follows: grade 0 , no lesion; grade 1, any lesion compressing only the thecal sac; grade 2 , any lesion in contact with the cord, without compression; grade 3, any lesion compressing the cord without signal change; and grade 4, any lesion compressing the cord with a signal change. Each grade was confirmed by the most severe lesion on the T2-weighted cervical and thoracic sagittal images, and a concurrent cervical or thoracic lesion was defined as a lesion showing more than grade 1 severity (Table 2, Fig. 1). The prevalence of concurrent cervical and

Table 2. Grade for severity of cervical or thoracic lesion

\begin{tabular}{cl}
\hline \hline Grade & Criteria \\
\hline 0 & No lesion \\
1 & Lesion compress the only thecal sac not spinal cord \\
2 & Lesion contact to the spinal cord with mild compression \\
3 & Lesion compress the spinal cord without signal change \\
4 & Lesion compress the spinal cord with signal change \\
\hline
\end{tabular}


thoracic lesions was then analyzed. In addition, the risk factors for a concurrent lesion, including gender, symptom duration and number of lumbar stenosis occurrences, were evaluated, and the relationship between the severity of the concurrent lesion and the same risk factors was separately analyzed. To determine the intraobserver and interobserver reliability, the severity of the lesion was graded twice with one-week intervals, and the dataset of the two different observers were compared and assessed.

\section{Results}

Seventy-seven patients (76.2\%) showed a concurrent cervical lesion and thirty patients (29.7\%) showed concurrent thoracic lesion. Twenty-six patients (25.7\%) showed both cervical and thoracic lesions, and 9 showed mild symptoms related to the concurrent lesion: mild axial pain in five, an intermittent tingling sensation of the upper extremities in four. However, there was no increase in the deep tendon reflex or myelopathic symptoms. Of the patients with concurrent cervical lesions, 56 had disc herniation, 17 had bony stenosis, and four had an ossified posterior longitudinal lig- ament (Table 3). In the thoracic spine, 18 patients had disc herniation and 12 had an ossified ligamentum flavum. The patients with concurrent cervical lesions showed the following lesion severity: grade 0, 24 patients; grade 1, 37 patients; grade 2, 16 patients; grade 3,21 patients; and grade 4, 3 patients. In the case of thoracic lesions, the

Table 3. Classification and severity of the concurrent cervical and thoracic lesion

\begin{tabular}{lrr}
\hline \hline & Cervical & Thoracic \\
\hline Classification of the lesion & & \\
Herniation of intervertebral disc & 56 & 18 \\
OPLL & 4 & 0 \\
OLF & 0 & 12 \\
Bony stenosis & 17 & 0 \\
Severity of the lesion & & \\
Grade 0 & 24 & 71 \\
Grade 1 & 37 & 19 \\
Grade 2 & 16 & 11 \\
Grade 3 & 21 & 0 \\
Grade 4 & 3 & 0 \\
\hline
\end{tabular}

OPLL: Ossification of posterior longitudinal ligament, OLF: Ossification of ligamentum flavum.
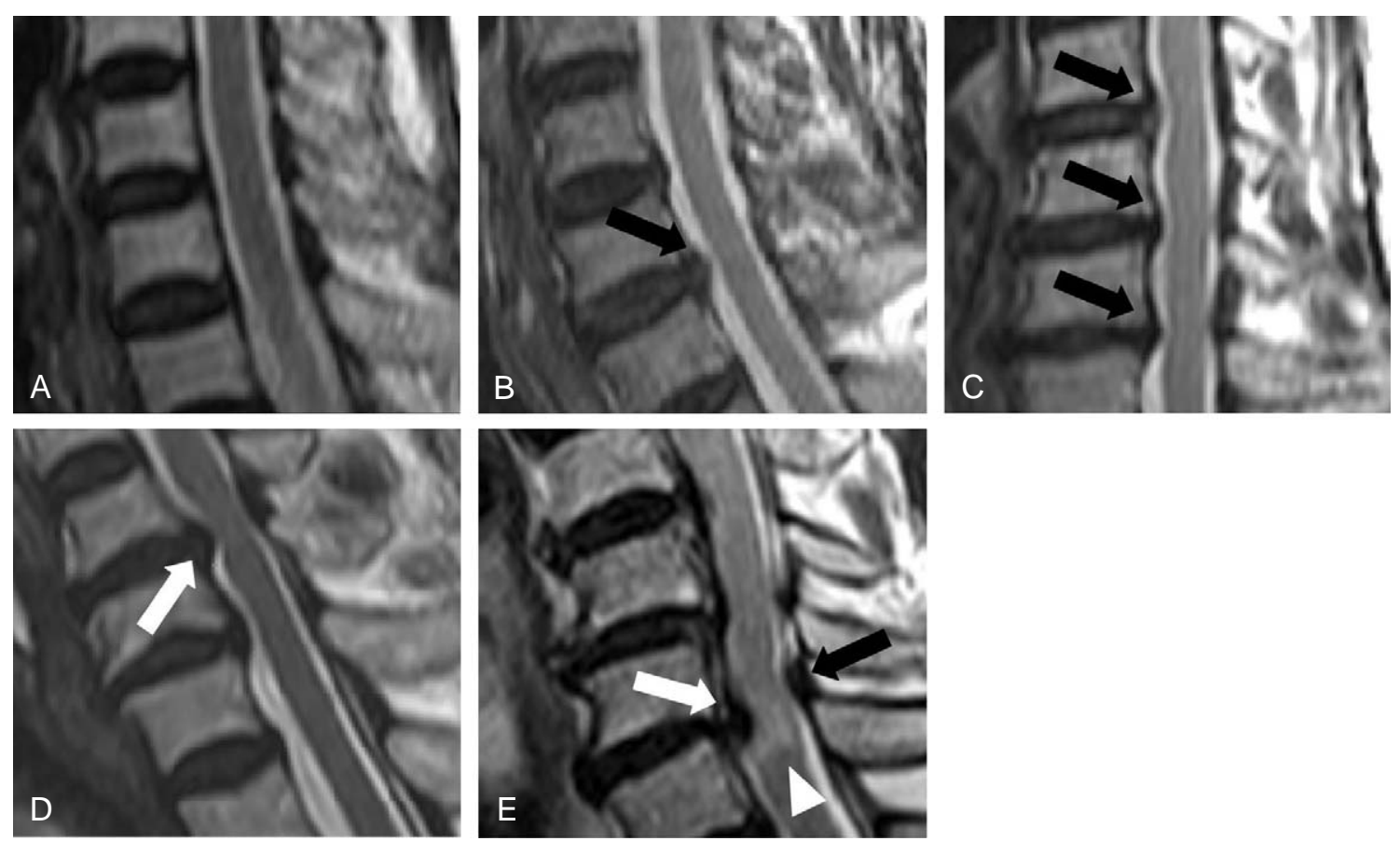

Fig. 1. Grades of the severity of cervical and thoracic lesion. (A) Grade 0: No compressive lesion. The cerebrospinal fluid (CSF) flow is intact, and there is no indentation with compression. (B) Grade 1: The herniated cervical intervertebral disc compresses the thecal sac (black arrow) but the CSF flow is maintained. (C) Grade 2: The herniated cervical intervertebral disc compresses the thecal sac and contacts the spinal cord with mild compression (black arrows). (D) Grade 3: The herniated cervical intervertebral disc compresses the spinal cord (white arrow) but there is no cord signal change. (E) Grade 4: The herniated cervical intervertebral disc (white arrow) and hypertrophic ligamentum flavum (black arrow) compress the spinal cord with a signal change (white arrowhead). 
degree of severity was graded as follows: grade 0,71 patients; grade 1, 19 patients; grade 2, 11 patients; grade 3, 0 patient; and grade 4, 0 patient.

The grading of the severity of the lesions showed high reliability. The interobserver reliability test showed the same consistency. The datasets that showed the highest reliability coefficient were used for data analysis (the second measurement of observer 2 was used). There was a positive correlation between the symptom duration of lumbar stenosis and the incidence of the cervical $(p=0.044)$ and thoracic $(p=0.022)$ lesions (Tables 4 and 5). However, there was no significant risk factor affecting the severity of the lesion in the concurrent cervical and thoracic lesions.

\section{Discussion}

The prevalence of a concurrent cervical lesion with no apparent symptoms in the patients in this study, who had undergone lumbar decompression with stenosis, was significantly high. The prevalence of thoracic lesions was much

Table 4. Risk factor of the patients had concurrent cervical lesion

\begin{tabular}{|c|c|c|c|}
\hline & Group I $(n=24)$ & Group II $(n=77)$ & $p$-value \\
\hline Mean age (yr) & $71.5(66-79)$ & $71.1(65-86)$ & $0.681^{\mathrm{a})}$ \\
\hline $65 \leq$ and $<70$ & 9 & 35 & $0.864^{\text {b) }}$ \\
\hline $70 \leq$ and $<80$ & 15 & 37 & \\
\hline$\geq 80$ & 0 & 5 & \\
\hline Sex ration (male/female) & $7 / 17$ & $32 / 45$ & $0.276^{c)}$ \\
\hline \multicolumn{4}{|l|}{ Level of lumbar stenosis } \\
\hline 1 level & 17 & 54 & $0.897^{\text {b) }}$ \\
\hline 2 level & 6 & 19 & \\
\hline 3 level & 1 & 4 & \\
\hline Mean Sx duration (mo) & $13.4(1.5-36)$ & $26.1(1.5-120)$ & $0.044^{\mathrm{d})}$ \\
\hline$<1(\mathrm{yr})$ & 15 & 29 & $0.010^{\mathrm{b})}$ \\
\hline $1 \leq \mathrm{Sx}$ duration $<3(\mathrm{yr})$ & 7 & 25 & \\
\hline $3 \leq \mathrm{Sx}$ duration $<5(\mathrm{yr})$ & 2 & 12 & \\
\hline$\geq 5$ (yr) & 0 & 11 & \\
\hline
\end{tabular}

Group I: Patients had no cervical lesion, Group II: Patients had concurrent cervical lesion.

Sx: Symptom.

${ }^{a}$ Independent two sample $t$-test, ${ }^{\text {b) }}$ Cochran-Mantel Haenszel test, ${ }^{\mathrm{c}}$ Chi-square test, ${ }^{\mathrm{d}}$ Wilcoxon rank-sum test.

Table 5. Risk factor of the patients had concurrent thoracic lesion

\begin{tabular}{|c|c|c|c|}
\hline & Group I $(n=71)$ & Group II $(\mathrm{n}=30)$ & $p$-value \\
\hline Mean age (yr) & $70.8(65-84)$ & $72.1(65-86)$ & $0.475^{\mathrm{a})}$ \\
\hline $65 \leq$ and $<70$ & 31 & 13 & $0.384^{\text {b) }}$ \\
\hline $70 \leq$ and $<80$ & 39 & 13 & \\
\hline$\geq 80$ & 1 & 4 & \\
\hline Sex ration (male/female) & $28 / 43$ & $11 / 19$ & $0.793^{\mathrm{c})}$ \\
\hline \multicolumn{4}{|l|}{ Level of lumbar stenosis } \\
\hline 1 level & 48 & 23 & $0.596^{\text {b) }}$ \\
\hline 2 level & 20 & 5 & \\
\hline 3 level & 3 & 2 & \\
\hline Mean Sx duration (mo) & $18.1(1.5-120)$ & $34.8(1.5-120)$ & $0.022^{\mathrm{a})}$ \\
\hline Sx duration $<1(\mathrm{yr})$ & 35 & 9 & $0.003^{\mathrm{b})}$ \\
\hline $1 \leq \mathrm{Sx}$ duration $<3(\mathrm{yr})$ & 23 & 9 & \\
\hline $3 \leq \mathrm{Sx}$ duration $<5(\mathrm{yr})$ & 10 & 4 & \\
\hline Sx duration $\geq 5$ (yr) & 3 & 8 & \\
\hline
\end{tabular}

Group I: Patients had no thoracic lesion, Group II: Patients had concurrent thoracic lesion.

Sx: Symptom.

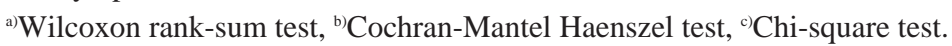


lower than that of the concurrent cervical lesion. However, simultaneously existing cervical and thoracic lesions were also detected in a number of patients. The longer symptom duration was related to the development of concurrent cervical and thoracic lesions. However, there was no factor that was related to the severity of the lesion.

Many studies reported the concurrent stenosis of the cervical and lumbar spine [3,6,12-15]. In 1984, Epstein et al. [1] showed that in a hospitalized cohort of patients with spinal stenosis, $5 \%$ had a symptomatic coexistent cervical and lumbar spinal stenosis. Dagi et al. [4] defined the symptom triad of the "symptomatic tandem spinal stenosis" as intermittent neurogenic claudication, progressive gait disturbance, and findings of mixed myelopathy and polyradiculopathy in both the upper and lower extremities. Previous clinical studies reported a 3.4-28\% incidence of tandem spinal stenosis [1,2,4,5,7]. Lee et al. [9] reported a $21.5 \%$ incidence of cervical stenosis and a $16.8 \%$ incidence of lumbar stenosis in their cadaveric study, as well as a $5.4 \%$ prevalence of tandem stenosis. They also reported a significantly higher prevalence of a combined lesion with cervical and lumbar stenosis than that of an isolated lumbar or cervical stenosis.

However, in previous clinical studies, only patients with a symptomatic concurrent cervical and lumbar stenosis were enrolled, and concurrent lesions with no apparent symptoms were not considered. Cadaveric studies also provided no information on the prevalence of stenosis in the soft tissues due to their use of cadaver skeletons, which also made it impossible to evaluate the relationship between the lesion and clinical symptoms [9]. In this study, $76.2 \%$ of the elderly subjects, who had undergone a decompressive procedure at the lumbar spine for stenosis, had cervical lesions. This is much higher than that reported in previous clinical studies, which evaluated symptomatic patients, suggesting that most elderly patients with a symptomatic lumbar stenosis had a concurrent cervical lesion. In addition, the prevalence of a concurrent thoracic lesion was $29.7 \%$, and $25.7 \%$ of patients had both cervical and thoracic lesions. These conditions have apparently higher incidences than previous studies.

Although concurrent stenosis accompanied by symptoms can create confusion in planning a treatment strategy, the development of diagnostic equipment, such as MRI, has made it easier to detect a concurrent lesion. Asymptomatic concurrent cervical or thoracic lesions in patients with symptomatic lumbar stenosis are difficult to recognize.
However, no study has reported the prevalence of asymptomatic concurrent cervical or thoracic lesion in patients with lumbar stenosis. Although there is no definitive report on the incidence of the postoperative aggravation of the symptoms due to an undetected concurrent spine lesion, such lesions can cause new symptoms to appear due to its aggravation by excessive flexion or extension posture of the cervical or thoracic spine during the intra- or operative period. Deem et al. [10] and Langmayr et al. [11] reported cases developing quadriplegia after thoracolumbar decompression in elderly patients with cervical spondylosis. They suggested that the cause of quadriplegia is cord compression by a hyperflexion posture or decreased cord blood supply as a result of positioning or hypotension. They made a point that careful positioning is essential in patients with cervical spondylosis when the prone position is used.

To evaluate the risk factors associated with the increased incidence of concurrent cervical and thoracic lesions, the authors analyzed the age at the time of surgery, gender, number of lumbar stenosis occurrences, and symptom duration. Among these, the symptom duration showed a significant correlation with both cervical and thoracic lesions. LaBan and Green [7] reported that a tandem stenosis is more frequent in patients older than 51 years of age. Although the present study enrolled elderly patients older than 65 years of age, there was no relationship between the concurrent lesion and age. In addition, no risk factors affecting the severity of the lesion were found.

This study had of the following limitations: (1) the possible intrinsic flaw of a retrospective study design, (2) foraminal stenosis was not considered because the diagnosis of concurrent stenosis was made only by a T2-weighted middle sagittal image, (3) the problematic evaluation of age as a risk factor because only patients over 65 years of age were enrolled, and (4) there was no control group for comparison purposes. Therefore, a further study of various age groups with a control group, as well as an evaluation of lumbar lesions in patients, who have undergone cervical or thoracic decompression for stenosis, will be needed.

\section{Conclusions}

Most of the elderly patients who had undergone lumbar decompressive surgery due to stenosis had a concurrent cervical lesion without apparent symptoms. A longer symptom duration was found to be related to a higher incidence of concurrent cervical and thoracic lesions. Therefore, to pre- 
vent unexpected clinical outcomes, it would be prudent to exclude concurrent lesions before commencing lumbar stenosis surgery in elderly patients.

\section{REFERENCES}

1. Epstein NE, Epstein JA, Carras R, Murthy VS, Hyman RA. Coexisting cervical and lumbar spinal stenosis: diagnosis and management. Neurosurgery 1984;15:489-96.

2. Hsieh CH, Huang TJ, Hsu RW. Tandem spinal stenosis: clinical diagnosis and surgical treatment. Changgeng Yi Xue Za Zhi 1998;21:429-35.

3. Teng P, Papatheodorou C. Combined cervical and lumbar spondylosis. Arch Neurol 1964;10:298-307.

4. Dagi TF, Tarkington MA, Leech JJ. Tandem lumbar and cervical spinal stenosis: natural history, prognostic indices, and results after surgical decompression. J Neurosurg 1987;66:842-9.

5. Aydogan M, Ozturk C, Mirzanli C, Karatoprak O, Tezer M, Hamzaoglu A. Treatment approach in tandem (concurrent) cervical and lumbar spinal stenosis. Acta Orthop Belg 2007;73:234-7.

6. Jacobs B, Ghelman B, Marchisello P. Coexistence of cervical and lumbar disc disease. Spine (Phila Pa 1976) 1990; $15: 1261-4$.

7. LaBan MM, Green ML. Concurrent (tandem) cervical and lumbar spinal stenosis: a 10-yr review of 54 hospitalized patients. Am J Phys Med Rehabil 2004;83:187-90.
8. Vogt MT, Cawthon PM, Kang JD, Donaldson WF, Cauley JA, Nevitt MC. Prevalence of symptoms of cervical and lumbar stenosis among participants in the Osteoporotic Fractures in Men Study. Spine (Phila Pa 1976) 2006;31: 1445-51.

9. Lee MJ, Garcia R, Cassinelli EH, Furey C, Riew KD. Tandem stenosis: a cadaveric study in osseous morphology. Spine J 2008;8:1003-6.

10. Deem S, Shapiro HM, Marshall LF. Quadriplegia in a patient with cervical spondylosis after thoracolumbar surgery in the prone position. Anesthesiology 1991;75:5278.

11. Langmayr JJ, Ortler M, Obwegeser A, Felber S. Quadriplegia after lumbar disc surgery: a case report. Spine (Phila Pa 1976) 1996;21:1932-5.

12. Chen SH, Hui YL, Yu CM, Niu CC, Lui PW. Paraplegia by acute cervical disc protrusion after lumbar spine surgery. Chang Gung Med J 2005;28:254-7.

13. Laroche M, Moulinier L, Arlet J, et al. Narrow cervical canal and lumbar canal: frequency of the association, role of hyperostosis. Rev Rhum Mal Osteoartic 1991;58:853-6.

14. Leroux JL, Legeron P, Moulinier L, et al. Stenosis of the lumbar spinal canal in vertebral ankylosing hyperostosis. Spine (Phila Pa 1976) 1992;17:1213-8.

15. Naderi S, Mertol T. Simultaneous cervical and lumbar surgery for combined symptomatic cervical and lumbar spinal stenoses. J Spinal Disord Tech 2002;15:229-31. 